\title{
the principle and implementation of blink of an eye detection and recognition for Humanoid type prosthetic
}

\author{
ZHENG Yi ${ }^{1, a}$, ZHOU Shufang ${ }^{2, b}, Y U E$ Qingchao ${ }^{2, c}$, DONG Li ${ }^{2, d}, Z H O N G$ Peisi ${ }^{1, e}$ \\ 1.Mechanical and electrical engineering, Shandong university of science and technology, Qingdao, \\ Shandong, 266590; \\ 2Mechanical and electrical engineering, Qingdao Huang hai University ,Qingdao, Shandong, \\ 266427
}

afw.2004@163.com,, $1349669548 @ q q . c o m,{ }^{\mathrm{c}}$ 121663260@qq.com, ${ }^{\mathrm{d}}$ 295792275@qq.com, ${ }^{\mathrm{e}} \mathrm{hhsjzhen}$ @163.com

Keywords: blink of an eye detection, Eye movement instrument, Humanoid type prosthetic, feature vectors

Abstract: Aiming at realizing the type prosthetic movement control problem to use the principle of blinking detection and recognition, the basic structure of a blink of an eye detection and recognition system was built, the basic principles of the blink of an eye detection and recognition was introduced, the whole process from wink event detection to identify control instruction was discussed, the blink of an eye detection and extraction of feature vector method has been carried by using Eye-movement apparatus combined with PC application, finally, the software testing effect was tested. Experimental results show that this method could detect blink signal at high accuracy and obtain feature vector, the purpose of the accurate control prosthetic limbs movement was achieved.

\section{Introduction}

According to statistics, The number of people with physical disability in China is 24.12 million, of which Twenty-nine percent were patients with upper limb disability. The movement of limbs has brought great inconvenience to their life. How to replace the missing limb with a machine, To make life easier for the disabled is an urgent problem to be solved in the field of rehabilitation medicine.

At present, The focus of prosthesis research is the selection of control source and control mode. Most prosthetic products in the market choose simple, easy to extract biological signals as their source of control, such as a signal of the muscles near the damaged joint. There are also some use of sound signals to complete control, but, the use of this kind of prosthesis is more serious and less practical. There are a few more advanced control methods, Using eeg or neural electrical signals to control, but, this method is still in the laboratory research stage [1].In the blink of an eye to control the prosthesis, the concealment is strong, the control is convenient, and to combine the blink events of different lengths, can produce a variety of different characteristics, Thus, more control functions are accomplished. 


\section{The basic principle of blinking detection and recognition}

The composition of the blink of an eye detection and recognition system

A blink detection and identification system, The five parts are composed of the eye image acquisition module, blinking detection module, feature extraction module, instruction identification module and instruction confirmation module. As shown in figure 1,

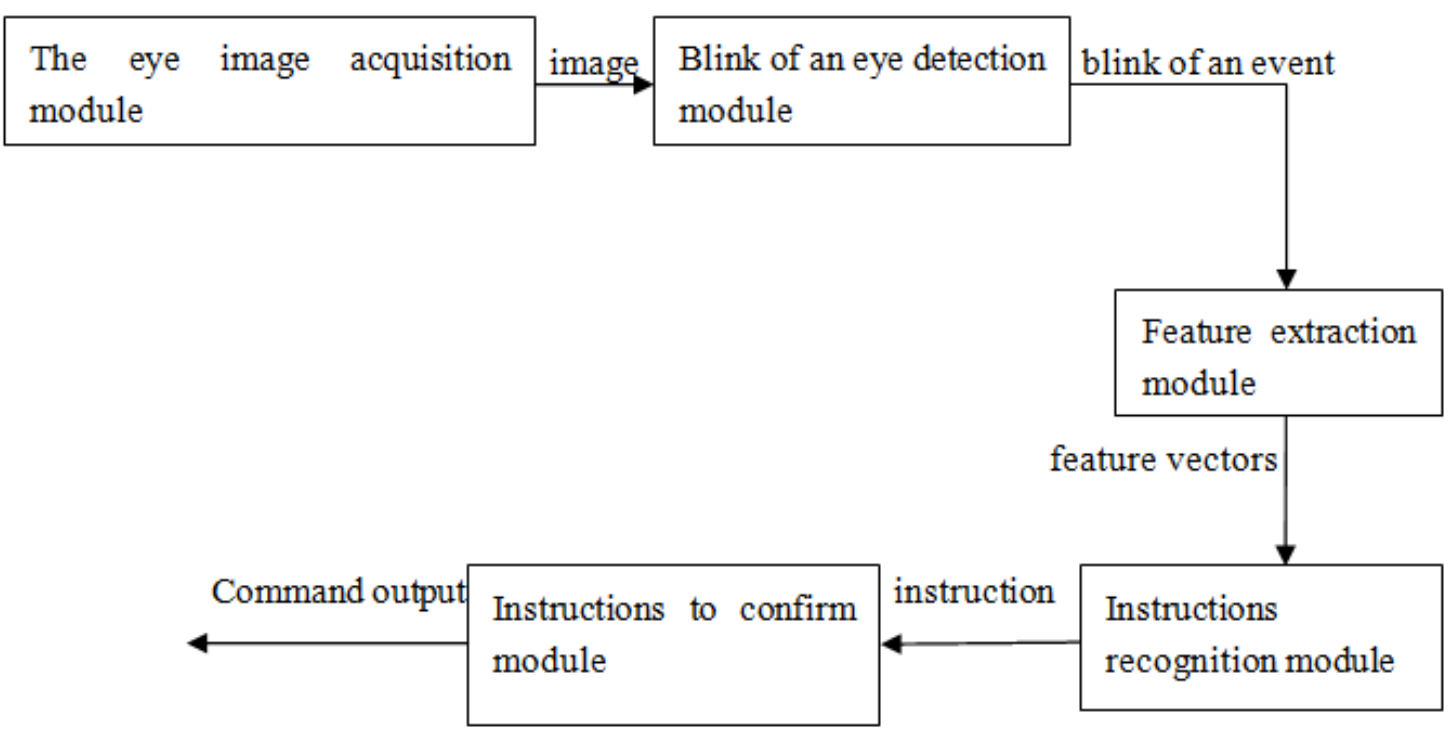

Fig.1. blink detection and recognition system

The two steps of detecting blinking and extracting feature vectors can be put together. Using the length of the duration to distinguish the blink event, firstly setting a time threshold $\mathrm{T}$ (for example, take $\mathrm{T}=0.7 \mathrm{~s}$ ). When you detect a blink event, record the closing time of the eye, To judge the length of the closing time, If the length is less than $\mathrm{T}$ and the number 0 is written to the eigenvector, writing the number 1 to the eigenvector if the length is greater than T. So if the test person blinks $\mathrm{n}$ times, the eigenvectors are stored in $\mathrm{n}$ Numbers in chronological order, each value is 0 or 1 depending on how long it takes to blink. If we say that the length of the eigenvector is 5 , the test's winking order is: The long blink (the closed eye length is greater than T), the short blink (the closed eye length is less than T), the long blink twice, the short blink once, The Numbers stored in the eigenvectors are: $[1,0,1,1,0]$.

To avoid error detection due to the test's natural blinking, May stipulate: The first element of the eigenvector formed by the blink of an eye must be 1 ,in other words, to produce an effective eigenvector, the first blink of an eye must be greater than $\mathrm{T}$.

In order to identify the motion instructions, the first step is to establish a one-to-one correspondence between eye movement eigenvectors and action commands. Let's say that there are 16 motion instructions associated with the movement of the prosthesis, so we need to have 16 eigenvectors that correspond to that, you can select the length of the eigenvector is 5,Remove the first element, and the other four elements can have 24 exactly 16 combinations. After extract the eigenvector, The program can use the lookup table method to find the corresponding instructions. 
The function of confirming the action instruction is to hand in the identified instructions to the tester for confirmation, according to the feedback information of the tester, the corresponding operation is conducted. The way to do this is: If the tester doesn't do anything in a given time, I think the instructions are correct, and output the instructions to the prosthetic control system, if the tester blinked twice in this period, the instruction is wrong and the instruction is cancelled.

To control the prosthesis in the blink of an eye, the test taker needs to remember the sequence of eye movements corresponding to the action command. In the control process, the tester blinked the eye in a certain order, and the corresponding control instructions were issued. Blink detection is used to identify the instructions to process is shown in figure 2 .

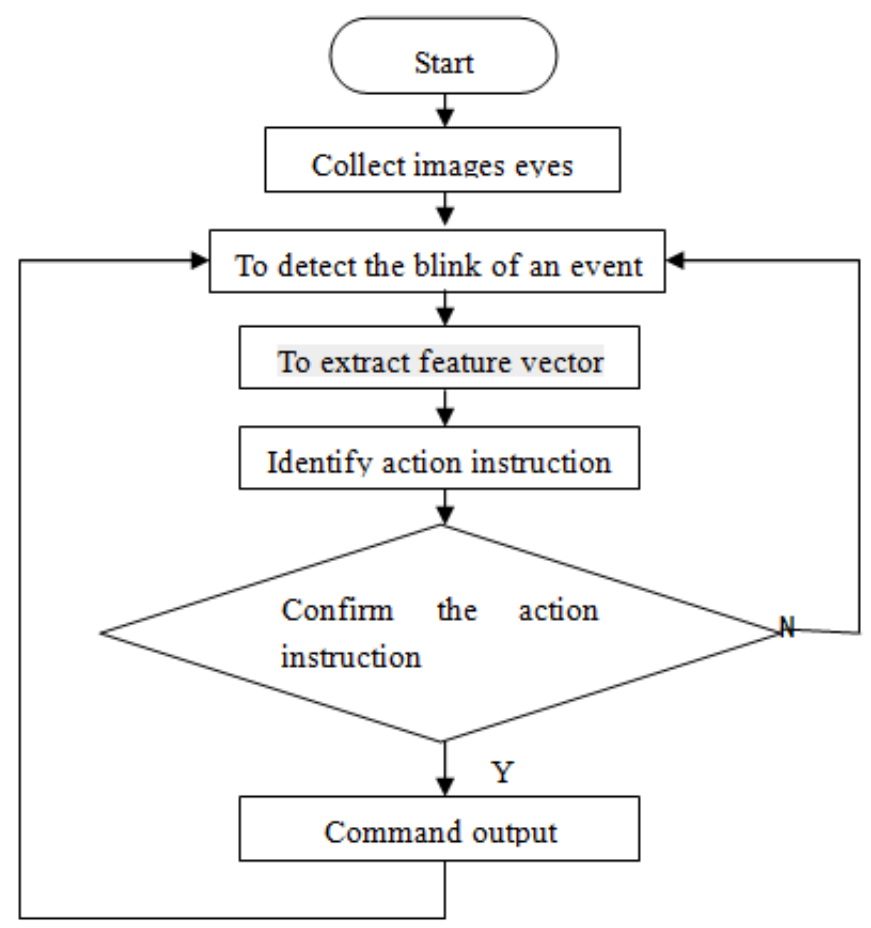

Fig. 2.The process of detecting blinking for command recognition

\section{Blink detection and recognition software implementation}

In this design, We use the existing SMI eye tracker and the upper computer software to complete the detection and recognition of blinking signals. The detection of eye image acquisition, processing and blink event was performed by SMI eye tracker. The upper computer software is developed by Visual c \#.

SMI eye movement instrument function

IView X series eye movement is designed and manufactured by German SMI company. Its working process is to illuminate the subject's eyes with infrared light. Infrared camera takes the video image of the subject's eyes. The output signal of the camera is sent to the computer for image data collection and analysis after MPEG coding. In real time, the horizontal and vertical motion of the eyes, the distance of the displacement, the velocity and the diameter of the pupil and the data of the fixation position were calculated. The shape of HED (head-mounted) eye movement is shown in figure.3: 


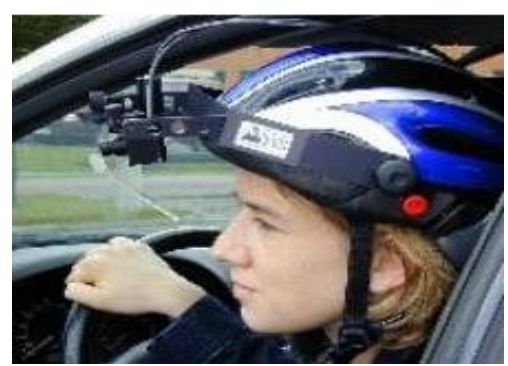

Fig.3. HED model (head-mounted) eye

Eye movement apparatus and application of network connection

In the design, we use the $\mathrm{c} \#$ development application and eye movement instrument iView $\mathrm{X}$ software running on the same PC, Therefore, the IP address of the data sender and receiver is the same, here are "127.0.0.1".Setting the sending end port number to "4444", and the receiver port number is set to "5555". Building a network connection can call the function provided by the iView X SDK:

public int iV_Connect(char[] sendIP, int sendPort, char[] receiveIP, int receivePort);

The parameter sendIP and sendPort is sending the address and port number, receiveIP and receivePort is address and port number at the receiving end.

Establishing a network connection can be implemented as follows:

public Eye Tracking Controller. Eye Tracking Controller ETDevice; Creating Eye Tracking Controller object:

string send ip = "127.0.0.1", send port $=$ "4444", receive ip = "127.0.0.1", receive port = "5555";

ETDevice.iV_Connect(sendip.ToCharArray(),Convert.ToInt32(sendport),receiveip.ToCharArra $\mathrm{y}()$, Convert.ToInt32(receive port)).

Blink of an eye detection and identification

The received data is EyeTrackingController SampleStruct structure as the basic unit.SampleStruct structures are defined as follows:

public struct SampleStruct

\{

public Int64 timestamp;

public EyeDataStruct leftEye, rightEye;

public int planeNumber;

\}

The timestamp, said the current time, the unit is 10-6 s;EyeDataStruct is the structure contains information about the eyes, there are two EyeDataStruct type in the SampleStruct, containing information for the right and left respectively.EyeDataStruct are defined as follows:

public struct EyeDataStruct 
public double gazeX; // The $\mathrm{X}$ position of the eye fixation position public double gazeY; // The Y position of the eye fixation position public double diam; // The pupil diameter public double eyePositionX; // The $\mathrm{x}$-coordinate of the eye position public double eyePositionY; // The y-coordinate of the eye position public double eyePositionZ; // \}

In the test, the closed eye time threshold is $\mathrm{T}=0.7 \mathrm{~s}$. The length of the eigenvector is 5 ,In addition to the first element must be 1 , the other four elements can be evaluated between 0 and 1 ,In this way, 24 = 16 action modes can be identified. In the program, for simplicity, we use a 5 bit binary $\mathrm{x}$ to represent the eigenvectors, Each of the elements in $\mathrm{x}$ correspond to each of the elements in the eigenvector. Also define a variable $\mathrm{n}$ to record the number of blinking. The procedure flow of detection algorithm is shown in figure 4:

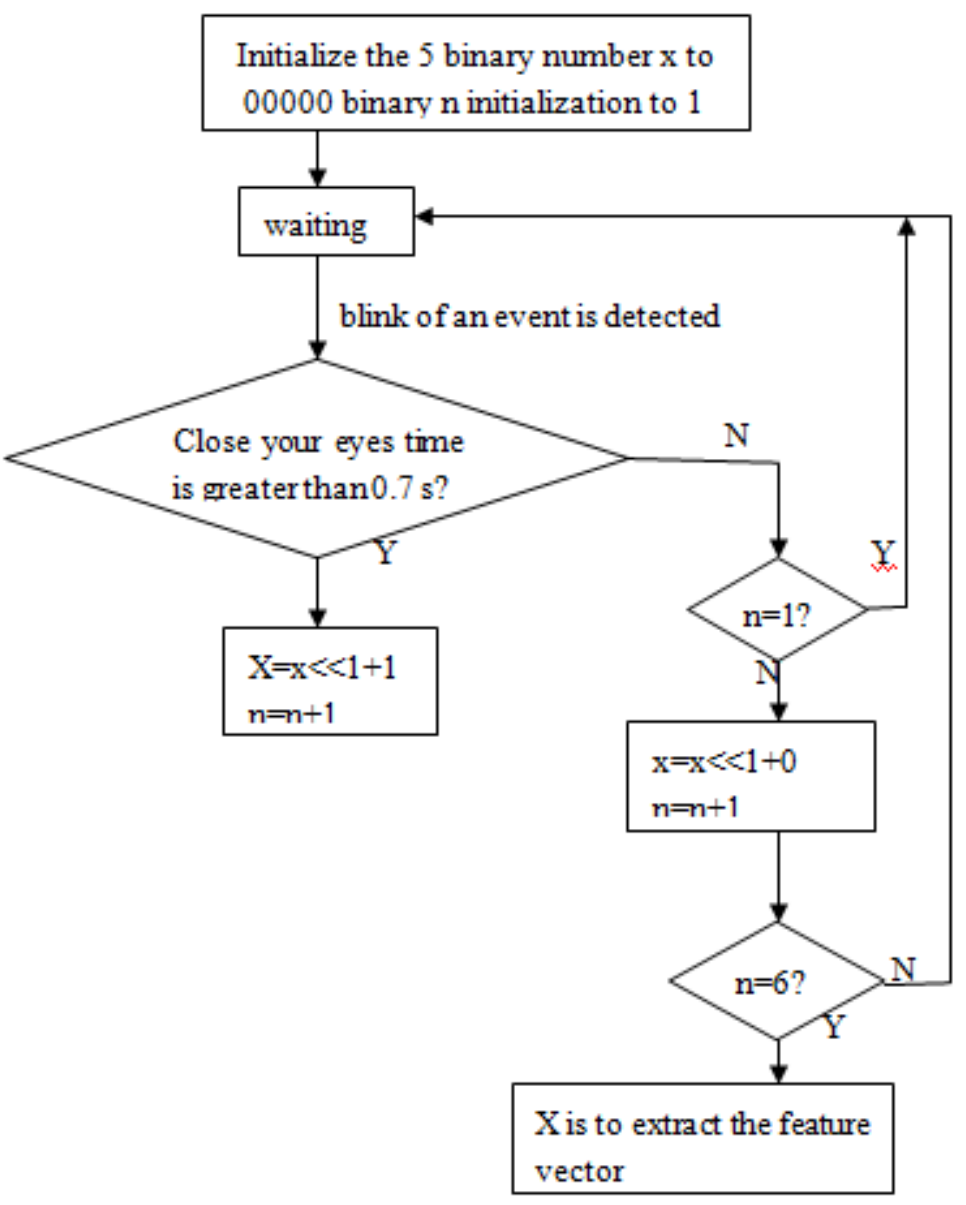

Fig. 4. blink detection algorithm flow chart

When the wearer blinked five times in a row, You can form an eigenvector, The premise is that the duration of the first blink is greater than 0.7.For example, when the tester blinked one time in chronological order and then blink four times, the output value of $\mathrm{x}$ was 10000 .

After the program detects the eigenvector, the corresponding control instructions are obtained according to the values of the eigenvectors. In this system design, The application that interacts 
with the iView $\mathrm{X}$ is responsible for detecting the blink event and getting the eigenvector $\mathrm{X}$, then sending $\mathrm{x}$ to the main control program of the prosthesis. The task of finding the corresponding control instructions by the value of $\mathrm{x}$ is completed by the main control program of the prosthesis.

\section{Detection effect test}

After a period of blinking at the tester, we tested the effect of blinking detection software. The tester blinked in 16 different sequences. Test 5 times in each order, and observe whether the output is the correct eigenvector. The test results are shown in table 1.

Table 1 blink detection software testing effect

\begin{tabular}{cccc}
\hline $\begin{array}{c}\text { The } \\
\text { feature }\end{array}$ & $\begin{array}{c}\text { Identifi } \\
\text { cation }\end{array}$ & $\begin{array}{c}\text { The } \\
\text { correct }\end{array}$ & $\begin{array}{c}\text { Wrong } \\
\text { number }\end{array}$ \\
vectors & number & number & \\
\hline 10000 & 5 & 5 & 0 \\
10001 & 5 & 5 & 0 \\
10010 & 5 & 5 & 0 \\
10011 & 5 & 5 & 0 \\
10100 & 5 & 5 & 0 \\
10101 & 5 & 5 & 0 \\
10110 & 5 & 5 & 0 \\
10111 & 5 & 5 & 0 \\
11000 & 5 & 5 & 1 \\
11001 & 5 & 5 & 0 \\
11010 & 5 & 5 & 0 \\
11011 & 5 & 5 & 0 \\
11100 & 5 & 4 & 1 \\
11101 & 5 & 5 & 0 \\
11110 & 5 & 5 & 0 \\
11111 & 5 & 5 & 0 \\
\hline & & &
\end{tabular}

The two errors of the corresponding eigenvector 11000 and 11100 are caused by the misoperation of the test subjects' eyes. The test results can be seen, as long as the tester memorized the blink sequence corresponding to the feature vector in advance, the software had a nearly $100 \%$ recognition rate. The identification time of a feature vector is approximately $4 \sim 5 \mathrm{~s}$. 


\section{Conclusion}

As a new upper limb control signal source, blinking signals have prominent advantages in controlling accuracy, anti-interference and concealment. The basic structure of a blink detection and recognition system is introduced, this paper discusses the whole process from detecting the blinking event to the extracting feature vector to the identification control instruction; the method of blinking detection and extraction of eigenvectors by the eye movement of SMI is presented; finally, the testing effect of software is tested. Experiments show that, the method used in this paper can detect the blinking signal with high accuracy and obtain the feature vector. the movement of prosthesis can be controlled accurately according to the feature vector recognition action instruction.

\section{Acknowledgment}

This work supported by Shandong University of science and technology plan projects (J16LB58); Graduate students of science and technology innovation projects(SDKDYC170221); National Natural Science Foundation of China (No. 51375282) and Project of Natural Science Foundation of Shandong Province, China (Grant No. ZR2015EM042).

\section{REFERENCE:}

[1]Hybrid speech recognition with deep bidirectional LSTM. GRAVES Alex, JAITLY Navdeep,MOHAMEDAbdel-rahman. 2013 IEEE Workshop on Automatic Speech Recognition and Understanding (ASRU) . 2013

[2]Large-scale, sequence-discriminative, joint adaptive training for masking-based robust ASR. NARAYANAN Arun, MISRA Ananya,CHIN Kean. 16th Annual Conference of the International Speech Communication Association (Interspeech 2015) . 2015

[3]Exploring multidimensional LSTMs for large vocabulary ASR. LI Jinyu, MOHAMED Abdelrahman, ZWEIG Geoffrey, et al. 2016 IEEE International Conference on Acoustics, Speech and Signal Processing (ICASSP) . 2016

[4]Long short-term memory recurrent neural network architectures for large scale acoustic modeling. Sak, Hasim,Andrew Senior,Francoise Beaufays. INTERSPEECH . 2014 [5]Conversational speech transcription using context-dependent deep neural netwoks. F.Seide,G.LI,D.Yu. Internation Speech Communication Association . 2011 [6] An investigation of deep neural networks for noise robust speech recognition. Seltzer ML, Yu D, Wang Y. 2013 IEEE International Conference on Acoustics,Speech and Signal Processing . 2013 [7]Underwater target estimation based on wideband laser-induced acoustic source. YE J M. . 2015 [8]Exploring convolutional neural network structures and optimization techniques for speech recognition. Abdel-Hamid O, Deng L, Yu D. Interspeech . 2013

[9]Deep convolutional neural networks for LVCSR. Sainath T N,Mohamed A R,Kingsbury B,et al. 2013 IEEE International Conference on Acoustics Speech and Signal Processing (ICASSP) . 2013 [10]Convolutional deep rectifier neural nets for phone recognition. Tóth L. Interspeech . 2013 [11]Speech recognition with deep recurrent neural networks. Graves A, Mohamed A-R, Hinton G. International Conference on Acoustics, Speech and Signal Processing . 2013

[12]"Long Short-Term Memory Recurrent Neural Network Architectures for Large Scale Acoustic Modeling,". H.Sak,A. Senior,F.Beaufays. INTERSPEECH . 2014 